\title{
Structure Map for Embedded Binary Alloy Nanocrystals
}

\author{
C. W. Yuan, ${ }^{1,2}$ S. J. Shin,,${ }^{1,2}$ C. Y. Liao, ${ }^{1,2}$ J. Guzman,,${ }^{1,2}$ P. R. Stone, ${ }^{1,2}$ \\ M. Watanabe, ${ }^{2}$ J. W. Ager III, ${ }^{2}$ E. E. Haller, ${ }^{1,2}$ and D. C. Chrzan ${ }^{1,2}$ \\ ${ }^{1}$ Department of Materials Science and Engineering, \\ University of California, Berkeley, CA 94720 \\ ${ }^{2}$ Materials Sciences Divison, Lawrence Berkeley National Laboratory, Berkeley, CA 94720
}

(Dated: November 7, 2008)

\begin{abstract}
The equilibrium structure of embedded nanocrystals formed from strongly segregating binaryalloys is considered within a simple thermodynamic model. The model identifies two dimensionless interface energies that dictate the structure, and allows prediction of the stable structure for any choice of these parameters. The resulting structure map includes three distinct nanocrystal morphologies: core/shell, lobe/lobe, and completely separated spheres.
\end{abstract}


The study of nanostructures embedded in a dielectric media has drawn much interest as their size-dependent thermal, optical and electrical behaviors suggest a wide range of potential applications.[1-5] Recently, attention has turned to the fabrication of embedded binary alloy nanostructures including core/shell [6-9] and lobe/lobe [10] nanostructures. Some synthesis routes (e.g., ion beam synthesis [6-9]) drive the system far from equilibrium, and often it is not clear if the experimentally observed structure is thermodynamically stable, or is the result of kinetics. However, knowledge of the stable structure is key to designing processing routes leading to desired nanostructures. There is thus a great need to understand the equilibrium morphology of embedded nanostructures.

There has been some theoretical work aimed at predicting the equilibrium morphology of embedded two-phase precipitates. Chatain et al. developed a theory for the morphology of a two-phase system embedded in a cavity with cubic symmetry [11] and presented a structure map indicating which morphologies are stable as a function of the relevant parameters. Mebed and Howe considered embedded lobe/lobe nanocrystals under the circumstance that the interface energy between the two phases is sharply cusped, so that this interface between the two phases in the cavity always remains flat.[12]

In this paper, a simple model describing the thermodynamic stability of embedded binaryalloy nanocrystals is discussed. The model considers elements A and B embedded within a matrix M. The compositions within each phase of the segregating alloy are assumed to be uniform. All interface free energies are assumed to be isotropic and all interfaces are assumed to be stress-free. The matrix is presumed to enable relaxation to the equilibrium shape. The free energies of competing structures are computed and compared, and the morphology minimizing the free energy is determined. Though the model is very simple, it enables a basic understanding of the obtainable structures, and provides a starting point for the development of more detailed models reflecting changes in composition, anisotropic interface energies, and the presence of interface and hydrostatic stresses.

Structures based on nanospheres are considered. For a eutectic A-B system, where $\alpha$ and $\beta$ are the A-rich and B-rich phases, four types of nanostructures are predicted to be possible: separated $\alpha$ and $\beta$ nanospheres [9, 10], $\alpha$-core $/ \beta$-shell or $\beta$-core $/ \alpha$-shell [7, 9], and $\alpha$-lobe/ $\beta$-lobe arrangements [10]. Within the assumptions given above, the equilibrium structure becomes a function of the volume fraction of the $\beta$-phase, $f_{\beta}$, and three interface free energies: $\gamma_{\alpha / M}, \gamma_{\beta / M}$ and $\gamma_{\alpha / \beta}$ where the subscripts indicate the relevant phases. ( $M$ is 
the matrix.)

The interfacial contributions to the free energies of the core/shell and separated sphere configurations are computed simply. Assuming that the total volume of the nanocrystal is $\frac{4 \pi}{3} R_{0}{ }^{3}$ and scaling all interfacial energies by $4 \pi R_{0}{ }^{2} \gamma_{\alpha / \beta}$ while defining $\gamma_{1}=\gamma_{\alpha / M} / \gamma_{\alpha / \beta}$ and $\gamma_{2}=\gamma_{\beta / M} / \gamma_{\alpha / \beta}$, one finds the (dimensionless) interfacial free energy for the $\alpha$-core $/ \beta$-shell phase, $E_{\alpha C / \beta S}$,

$$
E_{\alpha C / \beta S}=\left(1-f_{\beta}\right)^{2 / 3}+\gamma_{2}
$$

Similarly, the (dimensionless) interfacial free energy for the $\beta$-core $/ \alpha$-shell structure, $E_{\beta C / \alpha S}$, is

$$
E_{\beta C / \alpha S}=f_{\beta}^{2 / 3}+\gamma_{1}
$$

Finally, the (dimensionless) interfacial free energy for the separated spheres case, $E_{s e p}$, is given by:

$$
E_{\text {sep }}=\left(1-f_{\beta}\right)^{2 / 3} \gamma_{1}+f_{\beta}^{2 / 3} \gamma_{2}
$$

The lobe/lobe structure requires a more complicated analysis. Since all interfacial free energies are assumed to be isotropic, all the bounding surfaces are portions of spheres. The radii of curvature for the structures is then determined by the conditions for mechanical equilibrium of the triple point, and conservation of volume. A typical lobe/lobe structure is shown in Fig. 1, where the angles $\theta_{1}$ and $\theta_{2}$ are defined. The conditions for mechanical equilibrium of the triple point become (in dimensionless form):

$$
\gamma_{1} \cos \theta_{1}+\gamma_{2} \cos \theta_{2}=1
$$

and

$$
\gamma_{1} \sin \theta_{1}=\gamma_{2} \sin \theta_{2}
$$

Equations (4) and (5) completely determine the values of $\theta_{1}$ and $\theta_{2}$. Note that if any element of the triplet $\left\{1, \gamma_{1}, \gamma_{2}\right\}$ exceeds the sum of the other two, a mechanically stable triple point cannot exist and the lobe/lobe structure will not be stable.

Volume conservation is enforced by requiring the total volume of all structures to be $\frac{4 \pi}{3} R_{0}{ }^{3}$. The surface area and volume of a spherical cap are defined as

$$
A_{\text {cap }}(r, \phi)=2 \pi r^{2}(1-\cos \phi)
$$




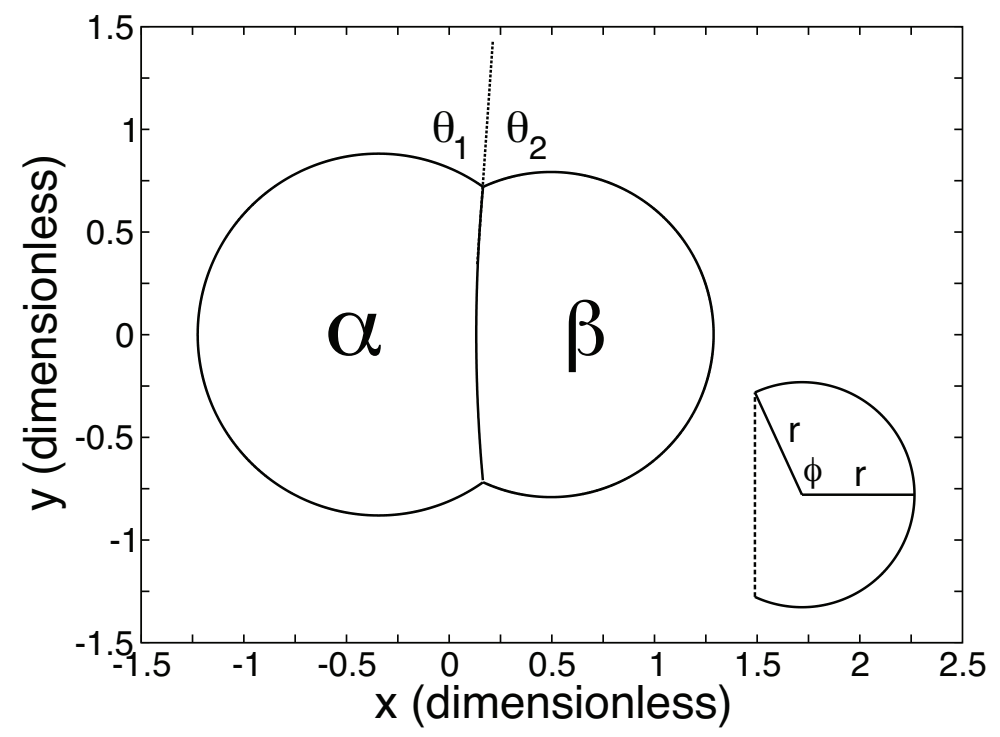

FIG. 1: Cross-sectional view of a lobe/lobe nanocrsytal configuration. $f_{\beta}=40 \%, \gamma_{1}=1.0$ and $\gamma_{2}$ $=1.0$. The dimensions are scaled by $R_{0}$, with $\frac{4 \pi}{3} R_{0}{ }^{3}=$ total volume of the structure. The contact angles $\theta_{1}$ and $\theta_{2}$ are shown, where the solid line is drawn tangent to the $\alpha / \beta$ interface at the triple point.

and

$$
V_{\text {cap }}(r, \phi)=\frac{2 \pi}{3} r^{3}(1-\cos \phi)-\frac{\pi}{3} r^{3} \cos \phi \sin ^{2} \phi,
$$

where $r$ is the radius of the sphere and $\phi$ is the polar angle characterizing the volume fraction of the cap (Fig. 1, inset) to the entire sphere. The total volume of the lobe/lobe structure therefore depends on two different pairs of $r$ and $\phi$, one for each spherical cap: $\alpha / M$ and $\beta / M$ (again, indicated by subscripts). The volume conservation equation for a lobe/lobe structure therefore goes as

$$
\begin{array}{r}
V_{\text {cap }}\left(r_{\alpha / M}, \phi_{\alpha / M}\right)+V_{\text {cap }}\left(r_{\beta / M}, \phi_{\beta / M}\right) \\
=\frac{4 \pi}{3} R_{0}{ }^{3} .
\end{array}
$$

Given $\gamma_{1}, \gamma_{2}$ and $f_{\beta}$, equations (4), (5), (7) and (8) together uniquely determine the geometry of the lobe-lobe structure. The total interfacial energy is then computed using Eq. 6. Eqs. (1), (2) and (3), when combined with the numerical solution for the lobe/lobe structure interfacial energy allow one to determine which of the four structures minimizes the interfacial free energy. Figure 2 shows free energy curves of the four possible structures 


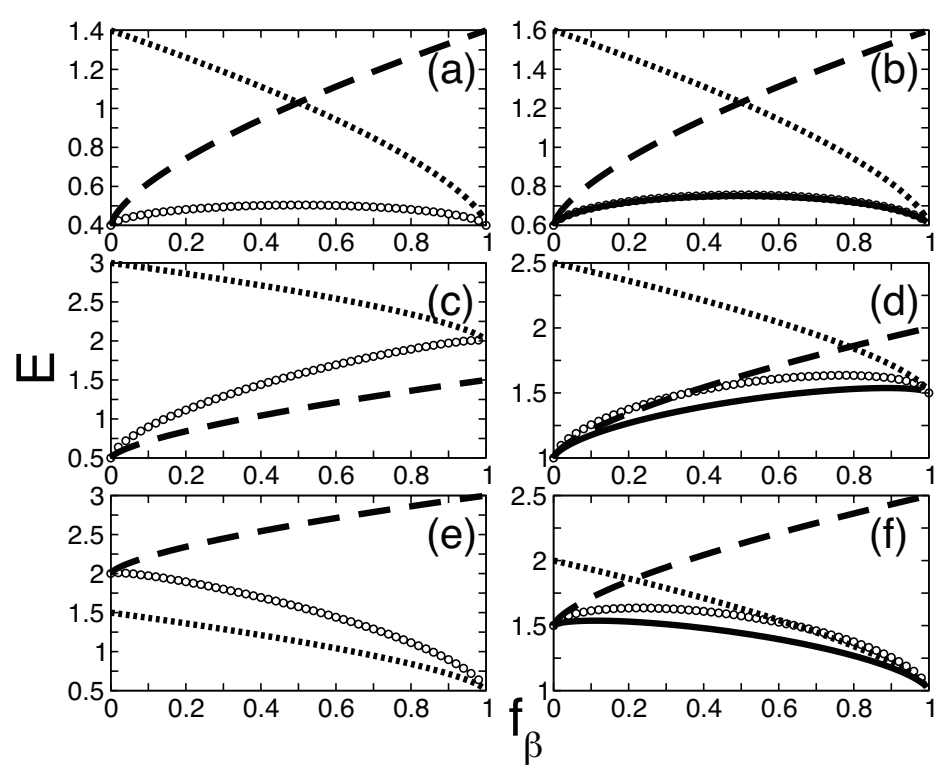

FIG. 2: Dimensionless free energy curves, as a function of $f_{\beta}$, for spherical nanocrystal configurations with $\left.\left\{\gamma_{1}, \gamma_{2}\right\}=\mathbf{a}\right)\{0.4,0.4\}$, b) $\{0.6,0.6\}$, c) $\{0.5,2.0\}$, d) $\{1.0,1.5\}$, e) $\{2.0,0.5\}$ and f) $\{1.5,1.0\}$. The dotted curve represents $\alpha$-core- $\beta$-shell, the broken line is $\beta$-core- $\alpha$-shell, the open circles represent two separate spheres and the solid line represents the lobe-lobe structure.

as a function of $f_{\beta}$, with different pairs of $\gamma_{1}$ and $\gamma_{2}$. Within our model, it is generally true that whenever a stable triple point can be defined, the lobe/lobe structure minimizes the interfacial free energy, irrespective of the value of $f_{\beta}$. Similarly, when a stable triple point cannot be defined, a unique structure minimizes the interfacial free energy for all values of $f_{\beta}$. Hence the equilibrium morphology of the embedded binary alloy nanocrystal is not affected by changes in volume fraction.

The fact that the stable morphology is independent of $f_{\beta}$ enables one to develop a simple structure map that holds for embedded nanocrystals. This structure map is presented in Fig. 3. The map consists of four regions. In the region for which $1>\gamma_{1}+\gamma_{2}$, separated spheres are stable. When $\gamma_{2}>1+\gamma_{1}, \alpha$-shells and $\beta$-cores are stable. In contrast, when $\gamma_{1}>1+\gamma_{2}, \beta$-shells and $\alpha$-cores are stable. For all other values of $\left(\gamma_{1}, \gamma_{2}\right)$, the lobe/lobe morphology is stable. Figure 3 also shows the progression of stable morphologies as one moves through parameter space for (a) $f_{\beta}=1 / 2$ and (b) $f_{\beta}=3 / 4$ for the nanocrystals. The lower left hand corner of each map shows how the structure changes when the boundary $\gamma_{1}+\gamma_{2}=1$ is crossed. The structures more centrally located in the map show how the 

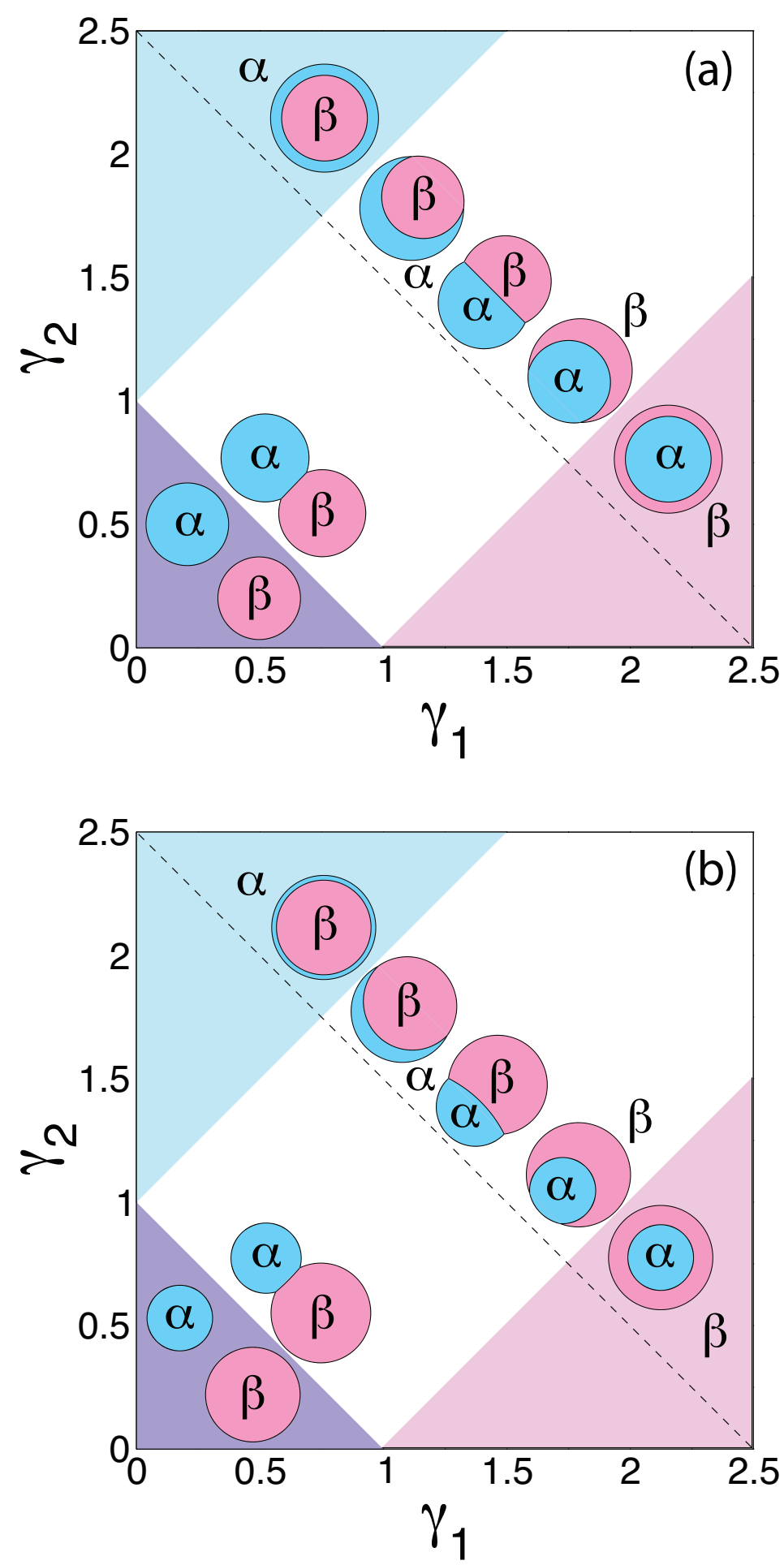

FIG. 3: Structural phase diagram for binary alloy nanocrystals with a) $f_{\beta}=0.5$ and b) $f_{\beta}=0.75$. The structures are located at the respective $\left(\gamma_{1}, \gamma_{2}\right)$ coordinates. 
morphology changes as one move along the line $\gamma_{1}+\gamma_{2}=2.5$, a line that passes through two boundaries on the structure map. Following the structures one sees how the core/shell structure yields to the lobe/lobe structure, how the lobe/lobe structure changes within its region of the structure map, and then reverses the role of core and shell materials upon passage through the final boundary.

The model thus yields a satisfyingly simple picture for the morphological stability of various nanostructures and should be useful as a "first-order" description of observed experimental results. For example, embedded interface energies are often sensitive to the presence of dopants [13]. Consequently, the structure map can serve as a tool for understanding how relative interface energies are altered by doping. One simply compares the observed morphologies for the doped and undoped cases to those in the structure map. Under optimal circumstances, this comparison can be used to guide the synthesis of desirable nanostructures.

This work is supported in part by the Directorate, Office of Science, Office of Basic Energy Sciences, Division of Materials Sciences and Engineering, of the U.S. Department of Energy under Contract No. DE-AC02-05CH11231 and in part by the US NSF grant No. DMR-0405472. 


\section{References}

[1] Q. Xu, I. D. Sharp, C. W. Yuan, D. O. Yi, C. Y. Liao, A. M. Glaeser, A. M. Minor, J. W. Beeman, M. C. Ridgway, J. W. Ager III, D. C. Chrzan, and E. E. Haller, Phys. Rev. Lett. 97, 155701 (2006).

[2] Y. Wang and H. Herron, J. Phys. Chem. 95, 525 (1991).

[3] J. Zheng, C. Zhang, and R. M. Dickson, Phys. Rev. Lett. 93, 077402 (2004).

[4] Y. M. Niquet, G. Allan, C. Delerue, and M. Lannoo, Appl. Phys. Lett. 77, 1182 (2000).

[5] J. D. Blauwe, IEEE Trans. Nano. 77, 1182 (2002).

[6] G. Mattei, Nucl. Instrum. Methods Phys. Res. B 191, 323 (2002).

[7] T. S. Anderson, R. H. M. III, J. E. Wiottig, D. L. Kinser, and R. A. Zuhr, Nucl. Instrum. Methods Phys. Res. B 171, 401 (2000).

[8] C. de Julian Fernandez, M. A. Tagliente, G. Mattei, C. Sada, V. Bello, C. Maurizio, G. Battaglin, C. Sangregorio, D. Gatteschi, L. Tapfer, and P. Mazzoldi, Nucl. Instrum. Methods Phys. Res. B 216, 246 (2004).

[9] P. Kluth, B. Hoy, B. Johannessen, S. G. Dunn, G. J. Foran, and M. C. Ridgway, Appl. Phys. Lett. 89 (2006).

[10] S. Shin (2007), unpublished research.

[11] D. Chatain, P. Wynblatt, S. Haǵege, E. J. Siem, and W. C. Carter, Interface Science 9, 191 (2001).

[12] A. M. Mebed and J. M. Howe, J. Mater. Sci. 42, 9504 (2007).

[13] S. Y. Chung, D. Y. Yoon, and S. J. L. Kang, Acta Materialia 50, 3361 (2002). 